\title{
The Use of Arm Tourniquet in Unilateral Carpal Tunnel Release: Does It Actually Have Advantage on Surgical Time \& Clinical Outcome?
}

\author{
Pyeong Soo Kim, Woo-Keun Kwon, Joo Han Kim, Youn-Kwan Park, Hong Joo Moon \\ Department of Neurosurgery, Korea University Guro Hospital, Korea University College of Medicine, Seoul, Korea
}

\begin{abstract}
Objective: To compare surgical time and operation preparing time, as well as clinical outcome of unilateral carpal tunnel release (CTR) between the groups which used tourniquet and those whom did not.

Methods: Of 53 cases of unilateral CTR under local anesthesia from 2011 to 2015, 35 were performed by using forearm tourniquets, while the other 18 were done without using tourniquets. The two groups were compared in terms of surgical time, total operating room (OR) time, preparing time and post-operative clinical outcomes by patients' satisfaction with the service.

Results: The mean surgical time was slightly shorter in cases performed with forearm tourniquets ( $31.63 \pm 6.86$ vs. $33.33 \pm$ $4.75 \mathrm{~min}, \mathrm{p}>0.05)$, however did not show any statistical significance. The mean total OR time ( $53.20 \pm 9.26$ vs. $51.22 \pm 7.03$ min, $p>0.05$ ) also did not show any statistically significant differences between groups. However, the mean preparing time for surgery was shorter in the no-tourniquet group with statistical significance $(17.89 \pm 4.44$ vs. $21.57 \pm 7.72 \mathrm{~min}, \mathrm{p}=0.03)$. All 53 patients revealed excellent or good surgical outcome, and there were no difference between two groups.

Conclusion: The use of forearm tourniquet in unilateral CTR operation under local anesthesia did not influence the actual surgical time nor the clinical outcomes, however did significantly reduce the preparing time for surgery. Therefore, considering the additional time needed for preparation without advantage on the surgical time or clinical outcomes, the use of tourniquet for this specific procedure is not optimal but optional, and can be selected following the surgeons preference.
\end{abstract}

Key Words: Carpal tunnel syndrome $\cdot$ Decompression $\cdot$ Operative time $\cdot$ Tourniquets

\section{INTRODUCTION}

The overall incidence of carpal tunnel syndrome (CTS) is known to be somewhat around $0.4 \%$ per year ${ }^{6}$. Carpal tunnel release (CTR) through a minimal invasive method such as endoscopic or classical open surgery both results in relatively goodto-excellent outcomes in more than $70 \%$ of the patients ${ }^{2)}$. CTR is one of the most commonly performed procedure in hand surgery ${ }^{10)}$.

CTR is most commonly performed as a day case procedure using local anesthesia and a tourniquet in many other centers ${ }^{8)}$ as well as ours. In hand surgery, a tourniquet is widely used to improve the view of the operative field while reducing blood loss. And for the same reason, is has been widely used by surgeons when performing CTR. However, some reports and clinical experiences suggest that upper limb pain, secon-

Corresponding author: Hong Joo Moon

Department of Neurosurgery, Korea University Guro Hospital, Korea

University College of Medicine, 148 Gurodong-ro, Guro-gu, Seoul 08308, Korea

Tel: +82-2-2626-3099, Fax: +82-2-863-1684

E-mail: vagusmoon@gmail.com dary to tourniquet use, is a common source of complaint for patients undergoing CTR under local anesthesia ${ }^{1)}$. This pain is not limited to intraoperative but also postoperative persistent complains sometimes.

To our knowledge, there has been some reports previously, studying about the surgical time or bleeding amount, but little has been reported regarding the actual operating surgical time or the preparing time before CTR using tourniquets. The aim of this study was to compare the results after using a forearm pneumatic tourniquet to those operated without the tourniquet, during surgical median nerve release at the carpal tunnel.

\section{MATERIALS AND METHODS}

Medical records of 5 men and 48 women aged 22 to 85 (mean, 59.23 \pm 9.59 ) years whom underwent open CTR at unilateral side between April 2011 and December 2015 were retrospectively reviewed. Only unilateral CTS patients were included because, regarding the preparing time for CTR, it could bring confusion in bilateral cases. Bilateral cases, revision cases, those with other underlying general diseases and those whom had CTR under general anesthesia were excluded. 
Of the 135 cases, 53 cases met the inclusion criteria. Of the 53 cases, 35 were operated by using the forearm tourniquet - tourniquet group by an experienced neurosurgeon $(\mathrm{JH}$ Kim), while the other 18 were operated without any tourniquets by another surgeon (HJ Moon) - non-tourniquet group. The neurosurgical team preparing the surgery were always the same. The outcomes in terms of overall operating room (OR) time, actual surgical time, preparing time for surgery, and patient satisfaction about the CTR (3-step scale from poor, good to excellent) were measured.

No patient received any sort of sedation or anxiolytics throughout the operation. Each patient at both groups had local anesthetic injections $(5-10 \mathrm{~mL}$ of $1 \%$ lidocaine) at the operating site, along the estimated line for intended incision. In the tourniquet group, a $60 \mathrm{~cm}$ long and $12 \mathrm{~cm}$ wide reusable tourniquet was applied to the forearm (Fig. 1). The tourniquet was inflated following elevation of the limb, skin preparation and whole arm draping. The tourniquet pressure was set at $250 \mathrm{mmHg}$ in all patients of this group. The non-tourniquet group did not set any tourniquets prior to operation.

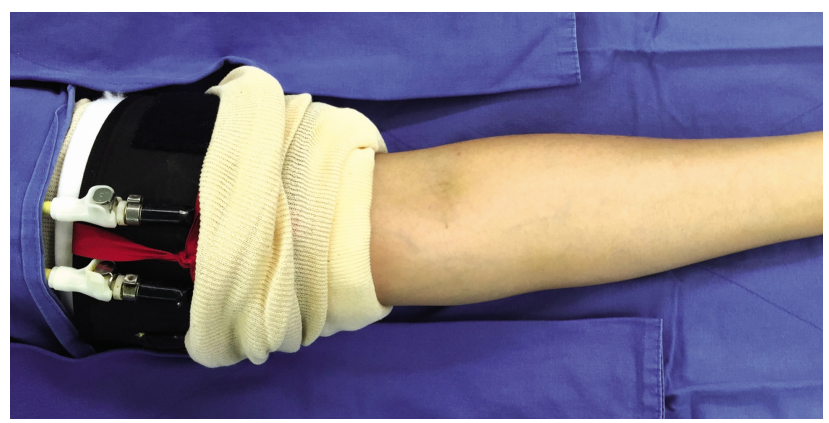

Fig. 1. Photograph of the forearm tourniquet used for unilateral carpal tunnel release. A $46 \mathrm{~cm}$ long and $10.5 \mathrm{~cm}$ wide reusable tourniquet is placed at the middle of the proximal arm with the pressure set at $250 \mathrm{mmHg}$ (A.T.S.TM 2000 Tourniquet System, Zimmer Orthopaedic Surgical Products, Dover, OH, USA).
Data are expressed as means \pm standard deviation (SD). The two groups were compared using the Chi-square test or independent student's $t$-test as appropriate. A p-value of less than 0.05 was considered to indicate statistical significance.

\section{RESULTS}

Demographic data of the patients and mean data of surgery related time, surgical outcome are shown on Table 1. There were no statistical difference regarding the mean age or the proportion of sex between two groups. The mean surgical time was slightly shorter in cases performed with forearm tourniquets ( $31.63 \pm 6.86$ vs. $33.33 \pm 4.75$ minutes, $p>0.05$ ), however did not show any statistical significance. The mean total OR time (53.20 \pm 9.26 vs. $51.22 \pm 7.03$ minutes, $\mathrm{p}>0.05$ ) also did not show any statistically significant differences between groups. However, the mean preparing time for surgery was shorter in the no-tourniquet group with statistical significance (17.89 \pm 4.44 vs. $21.57 \pm 7.72 \mathrm{~min}, \mathrm{p}=0.03)$. These differences are shown on Fig. 2 as a graph. All 53 patients revealed excellent or good surgical outcome, and there were no difference between two groups.

\section{DISCUSSION}

Since the invention of the pneumatic tourniquet by Cushing in 1908, the intraoperative tourniquet has not changed much until today ${ }^{5)}$ and hemostasis in hand surgery has traditionally been achieved by means of an arm tourniquet ${ }^{9}$. . However, even though complications related to use of pneumatic tourniquets are rare, its use is not completely without risk and its potential complications have been well documented in the literature $^{4,7,9)}$. They are mainly related to the tourniquet being inflated to an excessive pressure, or related to excessive dura-
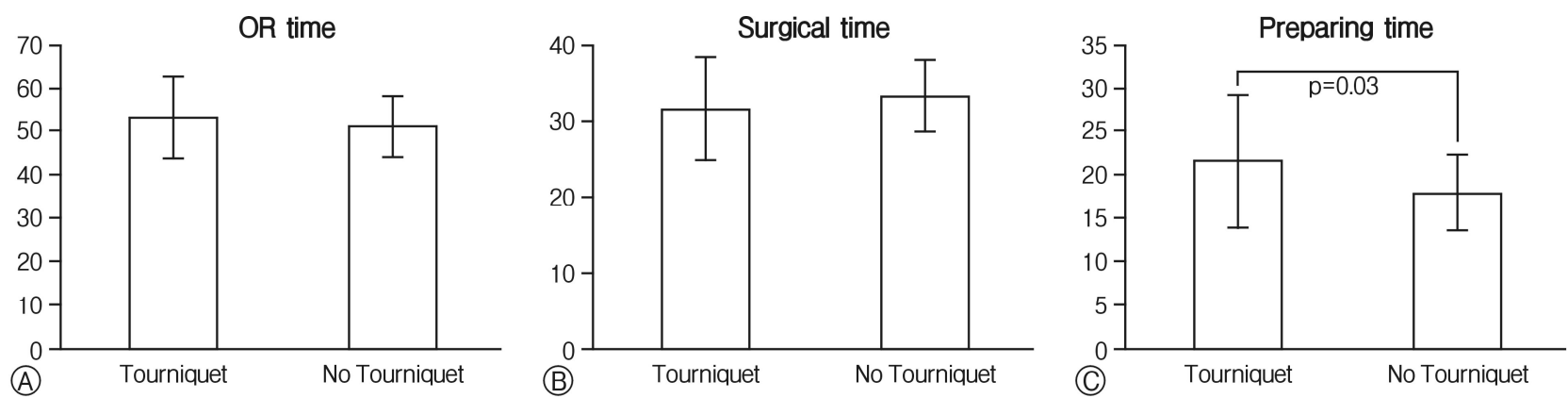

Fig. 2. Graph of the surgery related time in tourniquet group versus no-tourniquet group. (A) The mean surgical time was slightly shorter in cases performed with forearm tourniquets, but without any statistical significance. (B) The mean total operating room (OR) time also did not show any statistically significant differences between groups. (C) However, the mean preparing time for surgery was shorter in the no-tourniquet group with statistical significance $(p=0.03)$. 
Table 1. Demographic and surgery related data of unilateral carpal tunnel release

\begin{tabular}{lcc}
\hline \hline & Tourniquet group & No Tourniquet group \\
\hline Age & $54.11 \pm 9.96$ & $59.28 \pm 10.21$ \\
Sex & & \\
$\quad$ Male & $4(11.43 \%)$ & $1(5.56 \%)$ \\
Female & $31(88.57 \%)$ & $17(94.44 \%)$ \\
$\quad$ Total & 35 & 18 \\
Mean time & & \\
$\quad$ Operating room time & $53.20 \pm 9.26$ & $51.22 \pm 7.03$ \\
Surgical time & $31.63 \pm 6.86$ & $33.33 \pm 4.75$ \\
Preparing time & $21.57 \pm 7.72^{*}$ & $17.89 \pm 4.44^{*}$ \\
Surgical outcome & & \\
$\quad$ Excellent & $9(25.71 \%)$ & $6(33.33 \%)$ \\
Good & $26(74.29 \%)$ & $12(66.67 \%)$ \\
Poor & $0(0 \%)$ & $0(0 \%)$ \\
\hline *p-value $<0.05$ & &
\end{tabular}

${ }^{*}$ p-value $<0.05$

tion. These complications may include pain, nerve damage (intermittent or permanent sensory and/or motor paralysis), muscle damage (rhabdomyolysis), skin lesions (necrosis), and sometimes ischemia-reperfusion syndrome ${ }^{7}$.

Nevertheless, many surgeons including neurosurgeons still prefer the use of tourniquets during CTR because of the belief on possible hemostatic effect and because of the belief that the tourniquet might help reducing the surgical time, while the complication rate related tourniquets are quite low $^{7}$. However in our study, unlike the belief of some surgeons, we found that there was no significant decrease in the actual operating time nor in the OR time. Even more the use of tourniquet spent significantly more time for tourniquet preparation before surgery.

As mentioned once in the introduction, upper limb pain, secondary to tourniquet use, is a common source of complaint for patients undergoing CTR under local anesthesia ${ }^{3)}$. The mechanism of this intraoperative pain is complex. Besides other possible complications, arm pain is the most common complication, and it the one that bothers the patient most during surgery. Pain in the arm could also result in low compliance of the patient during local anesthesia, by moving the operating arm or other parts of the body due to pain. This can result in other indirect complications such as injury of the tissue which are not supposed to be manipulated during CTR. Therefore avoiding possible pain due to tourniquets might be also important, and could finally avoid other possible intraoperative problems.

Considering the facts mentioned above, it is doubtful if the tourniquet is really beneficial for unilateral CTR. As we presented in the results, the use of tourniquets significantly increased the preparing time for surgery, without any other benefits. No decrease of OR time or actual surgical time was achieved, there were no difference in clinical outcomes. Therefore considering the other possible complications of tourniquet use, most commonly, intraoperative arm pain, it seems we benefit not much from using tourniquets in CTR. Most importantly it did not influence the clinical outcome of CTR.

We still have some limitations in this study. The first to be mentioned is that the patients of compared two groups have undergone surgery by two different surgeons. The surgeon factor could have influenced the results of this study. However, as the operations were prepared by the same neurosurgical team, the preparing time for surgery which was one of the main points we evaluate in this article still remains meaningful. As the study was retrospective, we could not include the actual pain score or other objective vital signs of patients intraoperatively. So regarding the actual difference of pain between two groups, we are not able to state. But in terms of the time spent preparing the operation, the tourniquet does not seem to benefit. Another limitation is that, no difference in surgical time does not necessarily mean that there were no difference in the surgical view. Our study could have underestimated the benefit of tourniquet use in terms of surgical view, even when there were no difference in surgical time. Therefore, it remains reasonable for surgeons to select tourniquet use based upon their individual preference.

\section{CONCLUSION}

The use of forearm tourniquet in unilateral CTR operation under local anesthesia did not influence the actual surgical time nor the clinical outcomes, however did significantly increase the preparing time for surgery. Therefore, considering the additional time needed for preparation without advantage on the surgical time or clinical outcomes, the use of tourniquet for this specific procedure is not optimal but optional, and can be selected following the surgeons preference.

\section{DISCLOSURE}

No conflicts of interest were declared by the authors.

\section{REFERENCES}

1. Bidwai AS, Benjamin-Laing HE, Shaw DA, Iqbal S, Jones WA, Brown DJ: Patient satisfaction with tourniquet application and local anaesthesia infiltration in carpal tunnel decompression and the relationship with overall satisfaction. J Plast Surg Hand Surg 47:481-483, 2013 
2. Brown RA, Gelberman RH, Seiler JG, 3rd, Abrahamsson SO, Weiland AJ, Urbaniak JR, et al.: Carpal tunnel release. A prospective, randomized assessment of open and endoscopic methods. J Bone Joint Surg Am 75:1265-1275, 1993

3. Cousins GR, Gill SL, Tinning CG, Johnson SM, Rickhuss PK: Arm versus forearm tourniquet for carpal tunnel decompression - Which is better? A randomized controlled trial. J Hand Surg Eur Vol 40:961-965, 2015

4. Estebe JP, Mallédant Y: Pneumatic tourniquets in orthopedics. Ann Fr Anesth Reanim 15:162-178, 1996

5. Klenerman L: The tourniquet in surgery. J Bone Joint Surg Br 44B:937-943, 1962

6. Nordstrom DL, DeStefano F, Vierkant RA, Layde PM: Incidence of diagnosed carpal tunnel syndrome in a general population. Epidemiology 9:342-345, 1998

7. Odinsson A, Finsen V: Tourniquet use and its complications in Norway. J Bone Joint Surg Br 88B:1090-1092, 2006

8. Patil S, Ramakrishnan M, Stothard J: Local anaesthesia for carpal tunnel decompression: a comparison of two techniques. J Hand Surg Br 31:683-686, 2006

9. Ralte P, Selvan D, Morapudi S, Kumar G, Waseem M: Haemostasis in Open Carpal Tunnel Release: Tourniquet vs Local Anaesthetic and Adrenaline. Open Orthop J 4:234-236, 2010

10. Yiannakopoulos CK: Carpal ligament decompression under local anaesthesia: the effect of lidocaine warming and alkalinisation on infiltration pain. J Hand Surg Br 29:32-34, 2004 